\title{
Spontaneous Hemopericardium Leading to Cardiac Tamponade in a Patient with Essential Thrombocythemia
}

\author{
Anand Deshmukh, ${ }^{1,2}$ Shanmuga P. Subbiah, ${ }^{3}$ Sakshi Malhotra, ${ }^{4}$ Pooja Deshmukh, ${ }^{4}$ \\ Suman Pasupuleti, ${ }^{1}$ and Syed Mohiuddin ${ }^{1,4}$ \\ ${ }^{1}$ Department of Cardiovascular Medicine, Creighton University Medical Center, Omaha, NE 68131, USA \\ ${ }^{2}$ Creighton Cardiac Center, 3006 Webster Street, Omaha, NE 68131, USA \\ ${ }^{3}$ Department of Hematology and Oncology, Creighton University Medical Center, Omaha, NE 68131, USA \\ ${ }^{4}$ Department of Internal Medicine, Creighton University Medical Center, Omaha, NE 68131, USA
}

Correspondence should be addressed to Anand Deshmukh, drananddeshmukh@yahoo.com

Received 30 October 2010; Accepted 29 December 2010

Academic Editor: Syed Wamique Yusuf

Copyright (c) 2011 Anand Deshmukh et al. This is an open access article distributed under the Creative Commons Attribution License, which permits unrestricted use, distribution, and reproduction in any medium, provided the original work is properly cited.

Acute cardiac tamponade requires urgent diagnosis and treatment. Spontaneous hemopericardium leading to cardiac tamponade as an initial manifestation of essential thrombocythemia (ET) has never been reported in the literature. We report a case of a 72-year-old Caucasian female who presented with spontaneous hemopericardium and tamponade requiring emergent pericardiocentesis. The patient was subsequently diagnosed to have ET. ET is characterized by elevated platelet counts that can lead to thrombosis but paradoxically it can also lead to a bleeding diathesis. Physicians should be aware of this complication so that timely life-saving measures can be taken if this complication arises.

\section{Introduction}

Pericardial diseases principally manifest as pericarditis or pericardial effusion. Pericardial effusions resulting in cardiac tamponade can occur from a number of causes, including pericarditis, malignancy, acute myocardial infarction, endstage renal disease, congestive heart failure, collagen vascular disease, and viral and bacterial infections. Hemopericardium and resulting tamponade can result from any form of chest trauma, free wall rupture following myocardial infarction, retrograde bleeding into the pericardial sac following aortic root (type A aortic) dissection, as a complication of any invasive cardiac procedure, anticoagulation, and acute leukemias. In this paper, we describe a case of cardiac tamponade resulting from hemopericardium as an initial manifestation of essential thrombocythemia (ET).

\section{Case Presentation}

A 72-year-old Caucasian female presented to her primary care physician for ear pain. She was prescribed nonsteroidal anti-inflammatory drugs (NSAIDs) for her pain. A week later, she presented to the emergency department (ED) with episodes of chest pressure, worsening shortness of breath, and orthopnea. The patient had no known chronic medical illnesses in the past and was not on any other prescription medications. She had a 50-pack year history of smoking.

On physical examination, her heart rate was 115 beats/ minute and blood pressure was $123 / 44 \mathrm{~mm}$ of $\mathrm{Hg}$. Other vital signs were within normal limits. She had jugular venous distension on neck examination. Cardiovascular examination revealed tachycardia and positive pulsus paradoxus. Chest examination was unremarkable.

Laboratory data showed normal electrolytes and renal functions. Her white count was elevated at 12,900 cells/microliter, hemoglobin was marginally low at $11.2 \mathrm{~g} / \mathrm{dl}$, and platelet count was elevated at 745,000 cells/microliter. Cell differential was within normal limits. Cardiac biomarkers were within normal limits. TSH level was $3.57 \mathrm{mIU} / \mathrm{L}$. Electrocardiogram (ECG) showed normal sinus rhythm and left atrial enlargement. There were no electrical alternans or changes of pericarditis on the ECG. 
Chest X-ray showed cardiomegaly and small left sided pleural effusion. Computed tomography (CT) scan of the chest in the ED showed a moderate-sized pericardial effusion and a small left pleural effusion. Cardiology consultation was obtained due to the presence of pericardial effusion.

A transthoracic echocardiogram confirmed pericardial effusion with evidence of tamponade physiology (Figure 1). The patient was taken to cardiac catheterization laboratory and pericardiocentesis was done. A total of $300 \mathrm{~mL}$ of hemorrhagic fluid was drained during the initial procedure. Drainage of pericardial fluid resulted in immediate improvement of patient's symptoms. A pigtail catheter was left in place and the patient was transferred to coronary care unit. Over next two days, additional $310 \mathrm{~mL}$ of hemorrhagic fluid was drained and subsequently pigtail catheter was removed.

Pericardial fluid examination was unremarkable for any evidence of malignancy or infection. The etiology of hemorrhagic pericardial effusion was unclear even after extensive evaluation for malignancy, infection, autoimmune disease, or any other obvious systemic disease.

The patient continued to have elevated platelet count throughout the hospital stay with the highest platelet count being 855,000 cells/microliter. She denied any history of prior thrombotic or bleeding episodes. The elevation in platelet count was thought to be due to reactive thrombocytosis. The patient was discharged on fourth hospital day. She was instructed to followup with her primary care physician in two weeks with complete blood count to ensure resolution of thromobycytosis.

At followup, the patient continued to have elevated platelet counts. She was referred for hematology evaluation. A peripheral blood smear, bone marrow aspiration, and core biopsy were done for further evaluation of thrombocytosis. Peripheral blood smear showed occasional giant platelets with no platelet aggregates. There were no immature myeloid forms. Bone marrow aspirate showed prominent megaloblastoid change and increased number of megakaryocytes. Core biopsy also showed increased number of megakaryocytes. The megakaryocytes exhibited large hypersegmented nuclei with occasional megakaryocytic mitoses. Flow Cytometry was negative for any immunophenotypic abnormalities. Fluorescence in situ hybridization (FISH) for BCR-ABL translocation was negative arguing strongly against chronic myeloid leukemia. JAK 2 real-time PCR assay was negative for JAK2 V617 mutation. Thus, bone marrow biopsy showed megakaryocytic hyperplasia with dysmegakaryopoiesis consistent with ET.

\section{Discussion}

Pericardial effusions can be serous or hemorrhagic. The etiology of pericardial effusion is often influenced by the geographical region and patient-related factors. Hemorrhagic pericardial effusions often have different etiologies than serous effusions. In a retrospective study done by Atar et al., the most common cause of hemorrhagic effusions was found to be iatrogenic (31\%) namely, secondary to invasive cardiac procedures followed by malignancies [1].

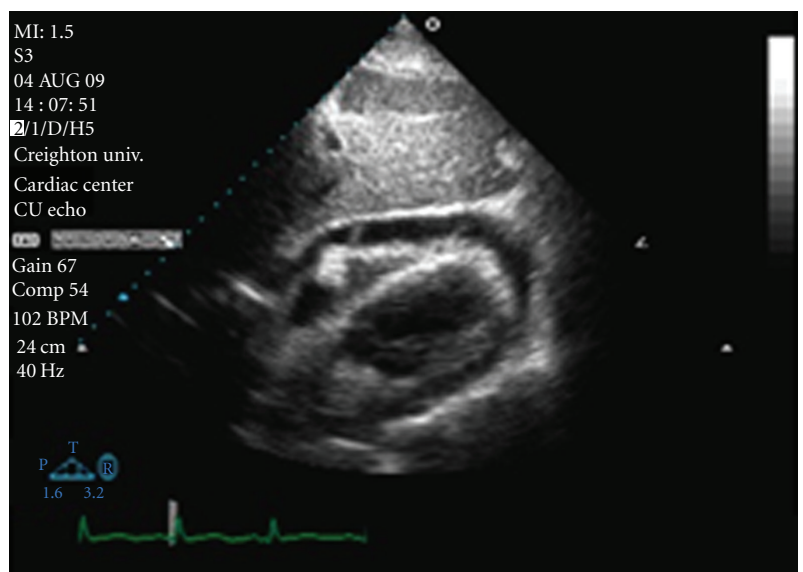

FIGURE 1: Subcostal view of hemorrhagic pericardial effusion. (There is a collapse of the right ventricular free wall during diastole.)

Bleeding diathesis leading to spontaneous hemopericardium secondary to congenital coagulation defect has been sparsely reported in the literature [2-4].

ET is currently classified among the $b c r / a b l$ negative, "Classic" myeloproliferative disorders (MPDs), which also include polycythemia vera (PV) and primary myelofibrosis (PMF) $[5,6]$. Each of these MPDs represents a stem cell derived clonal myeloproliferation. ET is characterized by megakaryocyte expansion leading to increased platelets. ET is an uncommon disease with prevalence of 2-3 cases per million. Median age at diagnosis is usually between 6575 years with female preponderance. The life expectancy of ET patients is generally long and similar to that of an agematched normal population [7]. Many patients with ET are asymptomatic, but a large number of them manifest symptoms or signs of bleeding, usually as ecchymoses or mucosal hemorrhage, or microvascular thrombosis, usually as erythromelalgia, digital ischemia, or transient ischemic attack $[8,9]$. The risk of thrombohemorrhagic complications remains unpredictable in individuals. The initial presentation in our patient was spontaneous hemopericardium that required emergency pericardiocentesis. Bleeding in ET is thought to be secondary to Acquired Von Willebrand disease (AVWD) and due to qualitative platelet defects. The main sites affected are skin, mucous membranes, and gastrointestinal tract. Severe bleeding is rare but fatal intracranial bleeding has been reported. AVWD in ET patients is characterized by the loss of large von Willebrand factor (VWF) multimers that results in a functional defect of the VWF, with increasing platelet counts (usually $>1.5$ million). Normalization of the platelet count results in normal plasma VWF multimeric distribution and regression of the hemorrhagic tendency [10]. Serious hemorrhages may be triggered by simultaneous antithrombotic therapy with anticoagulants, antiplatelet therapy, and NSAIDs. We believe that hemorrhagic conversion of pericardial effusion in our patient was triggered by NSAID therapy. However, we cannot exclude the possibility of viral syndrome resulting in pericarditis with subsequent conversion to hemorrhagic 
pericardial effusion from bleeding complications related to ET. Kayrak et al. reported a similar case of a patient with essential thrombocythemia treated with acetylsalicylic acid, which resulted in hemorrhagic pericardial effusion [11]. The patient was successfully treated with clopidogrel therapy without any hemorrhagic or thrombotic event. Averback and Moinuddin describe a case of pericardial effusion secondary to essential thrombocythemia [12]. However, the patient had trilineage hyperplasia and authors describe autopsy features consistent with agnogenic myeloid metaplasia with predominant megakaryocytic proliferation. The diagnosis of ET on the basis of current WHO criteria is questionable in this case. Thus, we believe that our case is the first one of ET manifesting with spontaneous hemopericardium as the initial manifestation. Also, the diagnosis of ET in our patient was made based on WHO criteria [13]. In ET, bone marrow findings are remarkable for the presence of large, but mature-appearing, megakaryocytes with deeply lobulated and hyperlobulated nuclei that are most often dispersed throughout the biopsy sections [14]. Our patient did have morphological megakaryocyte abnormality consistent with diagnosis of ET.

Pericardial effusion related to ET should be treated based on current standards of care. Patients with cardiac tamponade require urgent pericardiocentesis. Bleeding complications may be prevented by avoiding use of high-dose aspirin and NSAIDs. In patients with bleeding complications related to ET and AVWD, treatment of thrombocytosis may be considered [15]. Patients with ET requiring antiplatelet therapy can be treated with clopidogrel therapy [11].

To summarize, spontaneous hemopericardium is infrequently encountered among patients with ET, and its clinical recognition may be difficult. Therefore, in patients with ET who manifest acute dyspnea or clinical signs of hemodynamic embarrassment, hemopericardium and tamponade should be ruled out. Pericardiocentesis is life saving in these patients since their clinical course is otherwise relatively benign.

\section{References}

[1] S. Atar, J. Chiu, J. S. Forrester, and R. J. Siegel, "Bloody pericardial effusion in patients with cardiac tamponade. Is the cause cancerous, tuberculous, or latrogenic in the 1990s?" Chest, vol. 116, no. 6, pp. 1564-1569, 1999.

[2] L. W. Gaston, B. F. Mach, and W. S. Beck, "Hemophilia A and concurrent factor VII deficiency. Studies of a patient with complicating cardiac tamponade," The New England Journal of Medicine, vol. 264, pp. 1078-1082, 1961.

[3] G. A. Anderson, "Spontaneous hemopericardium with cardiac tamponade and "pericardiotomy syndrome" complicating hemophilia," The American Journal of Cardiology, vol. 13, no. 2, pp. 278-283, 1964.

[4] S. C. Schultz, J. Breall, and R. Hannan, "Acute cardiac tamponade secondary to congenital factor V deficiency," Cardiology, vol. 88, no. 1, pp. 48-49, 1997.

[5] A. Tefferi and T. Barbui, "bcr/abl-negative, classic myeloproliferative disorders: diagnosis and treatment," Mayo Clinic Proceedings, vol. 80, no. 9, pp. 1220-1232, 2005.
[6] P. J. Campbell and A. R. Green, "The myeloproliferative disorders," New England Journal of Medicine, vol. 355, no. 23, pp. 2452-2466, 2006.

[7] C. Rozman, M. Giralt, E. Feliu, D. Rubio, and M. T. Cortes, "Life expectancy of patients with chronic nonleukemic myeloproliferative disorders," Cancer, vol. 67, no. 10, pp. 2658-2663, 1991.

[8] M. Colombi, F. Radaelli, L. Zocchi, and A. T. Maiolo, "Thrombotic and hemorrhagic complications in essential thrombocythemia: a retrospective study of 103 patients," Cancer, vol. 67, no. 11, pp. 2926-2930, 1991.

[9] S. Cortelazzo, P. Viero, G. Finazzi, A. D’Emilio, F. Rodeghiero, and T. Barbui, "Incidence and risk factors for thrombotic complications in a historical cohort of 100 patients with essential thrombocythemia," Journal of Clinical Oncology, vol. 8, no. 3, pp. 556-562, 1990.

[10] U. Budde and P. J. J. Van Genderen, "Acquired von Willebrand disease in patients with high platelet counts," Seminars in Thrombosis and Hemostasis, vol. 23, no. 5, pp. 425-431, 1997.

[11] M. Kayrak, K. Acar, M. Yazici, C. Kaya, S. Selim Ayhan, and H. Gok, "Pericardial hemorrhage due to acetylsalicylic acid in a patient with essential thrombocythemia," Clinical and Applied Thrombosis/Hemostasis, vol. 15, no. 4, pp. 465-466, 2009.

[12] P. Averback and M. Moinuddin, "Pericarditis as a manifestation of essential thrombocythemia," Canadian Medical Association Journal, vol. 117, no. 2, pp. 154-156, 1977.

[13] J. W. Vardiman, J. Thiele, D. A. Arber et al., "The 2008 revision of the World Health Organization (WHO) classification of myeloid neoplasms and acute leukemia: rationale and important changes," Blood, vol. 114, no. 5, pp. 937-945, 2009.

[14] J. Thiele, H. M. Kvasnicka, V. Diehl, R. Fischer, and J. J. Michiels, "Clinicopathological diagnosis and differential criteria of thrombocythemias in various myeloproliferative disorders by histopathology, histochemistry and immunostaining from bone marrow biopsies," Leukemia and Lymphoma, vol. 33, no. 3-4, pp. 207-218, 1997.

[15] U. Budde, G. Schaefer, and N. Mueller, "Acquired von Willebrand's disease in the myeloproliferative syndrome," Blood, vol. 64, no. 5, pp. 981-985, 1984. 


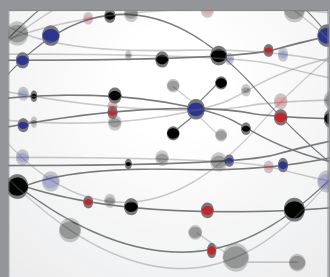

The Scientific World Journal
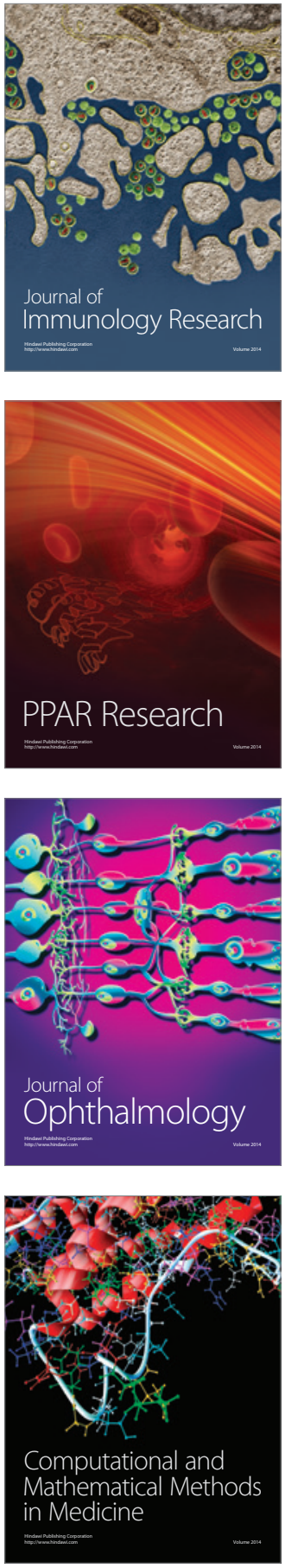

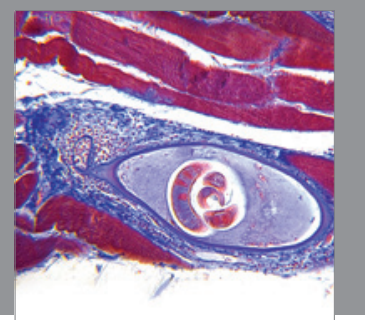

Gastroenterology

Research and Practice
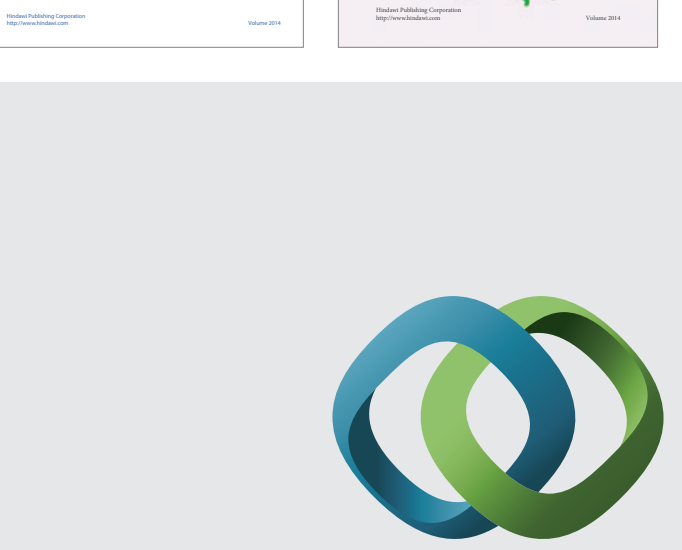

\section{Hindawi}

Submit your manuscripts at

http://www.hindawi.com
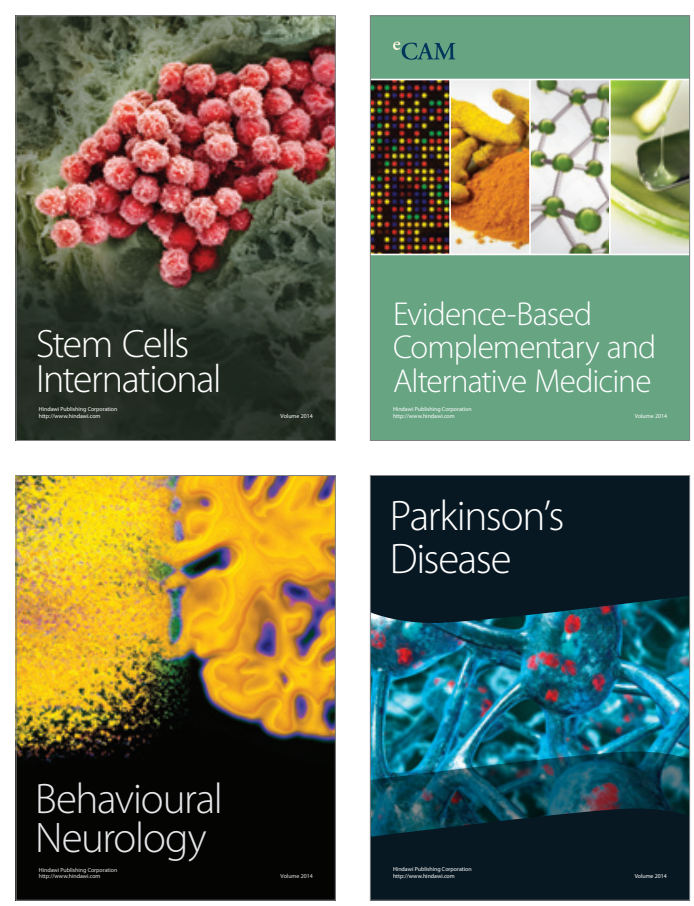

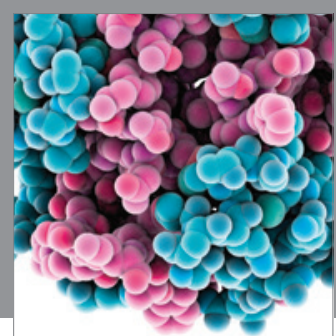

Journal of
Diabetes Research

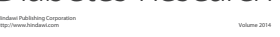

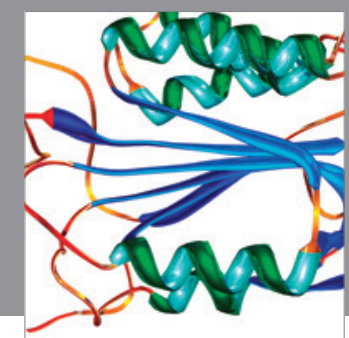

Disease Markers
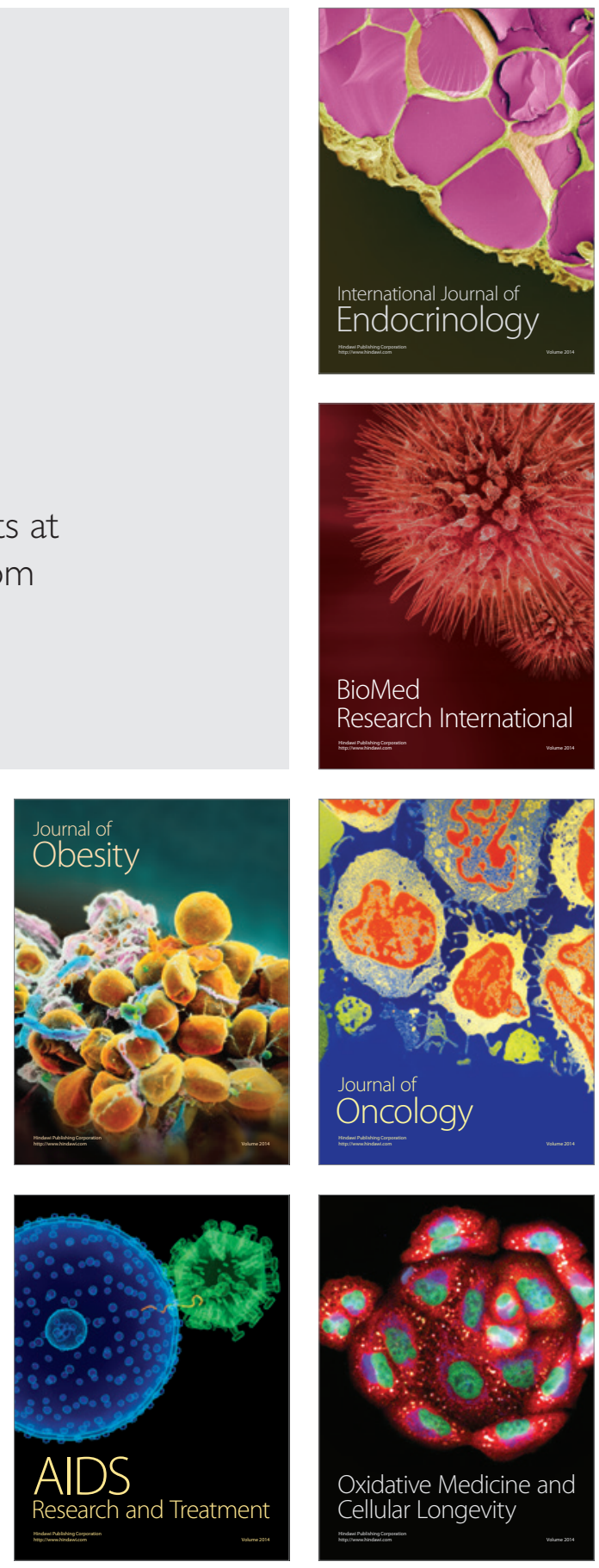